\title{
Association Between Anxiety and Depression and Gastroesophageal Reflux Disease: Results From a Large Cross-sectional Study
}

\author{
Ji Min Choi, ${ }^{1}$ Jong In Yang, ${ }^{1}$ Seung Joo Kang, ${ }^{\text {* }}$ Yoo Min Han, ${ }^{1}$ Jooyoung Lee, ${ }^{1}$ Changhyun Lee, ${ }^{1}$ Su Jin Chung, ${ }^{1}$ Dae Hyun Yoon, ${ }^{2}$ \\ Boram Park, ${ }^{1}$ and Yong Sung Kim ${ }^{3}$ \\ ${ }^{I}$ Department of Internal Medicine and Healthcare Research Institute, Healthcare System Gangnam Center, Seoul National University Hospital, \\ Seoul, Korea; ${ }^{2}$ Department of Psychiatry, Healthcare System Gangnam Center, Seoul National University Hospital, Seoul, Korea; and ${ }^{3}$ Division of \\ Gastroenterology, Department of Internal Medicine, Wonkwang University Sanbon Hospital, Gunpo, Gyeonggi-do, Korea
}

\section{Background/Aims \\ The different clinical manifestations of gastroesophageal reflux disease (GERD) may be influenced by associated psychological factors. We evaluated the psychological status (anxiety and depression) according to each subtype of GERD. \\ Methods \\ Subjects who underwent esophagogastroduodenoscopy and completed a symptom questionnaire between January 2008 and December 2011 were analyzed. The subjects were classified into the following groups: erosive reflux disease (ERD), non-erosive reflux disease (NERD), asymptomatic erosive esophagitis (AEE), and controls. Anxiety and depression were assessed using the State-Trait Anxiety Inventory and Beck Depression Inventory, respectively.}

\section{Results}

We analyzed 19099 subjects: $16157(84.6 \%), 176(0.9 \%), 1398$ (7.3\%), and 1368 (7.2\%) in the control, ERD, NERD, and AEE groups, respectively. Multiple multinomial logistic regression revealed a significant association of increased state (adjusted OR, 1.89; $95 \% \mathrm{Cl}, 1.53-2.33$ ) and trait anxiety (adjusted OR, 1.78; 95\% Cl, 1.34-2.35) and depression (adjusted OR, 2.21; 95\% Cl, 1.75-2.80) with NERD. ERD group showed a significant association only with state anxiety (adjusted OR, 2.20; 95\% Cl, 1.27-3.81) and depression (adjusted OR, 2.23; 95\% Cl, 1.18-4.22). The AEE group, however, did not show any significant association with psychological factors.

\section{Conclusion}

This cross-sectional study revealed that anxiety and depression levels were significantly higher in subjects with GERD (notably in the NERD) than in controls.

(J Neurogastroenterol Motil 2018;24:593-602)

\section{Key Words}

Anxiety; Depression; Gastroesophageal reflux; Psychology

Received: April 6, 2018 Revised: June 18, 2018 Accepted: August 10, 2018

(a) This is an Open Access article distributed under the terms of the Creative Commons Attribution Non-Commercial License (http://creativecommons. org/licenses/by-nc/4.0) which permits unrestricted non-commercial use, distribution, and reproduction in any medium, provided the original work is properly cited.

${ }^{*}$ Correspondence: Seung Joo Kang, MD

Department of Internal Medicine and Healthcare Research Institute, Healthcare System Gangnam Center, Seoul National University Hospital, GFC Building 152, Teheran Ro, Gangnam-gu, Seoul 06236, Korea

Tel: +82-2-2112-5772, Fax: +82-2-2112-5635, E-mail: ksjoo55@naver.com

Ji Min Choi and Jong In Yang contributed equally to this paper. 


\section{Introduction}

Gastroesophageal reflux disease (GERD) is defined as a condition in which gastric contents reflux to the esophagus, causing troublesome symptoms such as heartburn and acid regurgitation. ${ }^{1}$ GERD is one of the most prevalent gastrointestinal disorders globally, showing an increasing prevalence in several developing countries. $^{2,3}$ This disorder runs a chronic course, leads to a considerable deterioration in the quality of life of patients, and is associated with a high economic burden worldwide. ${ }^{4-6}$ Furthermore, in a few patients, GERD may progress and cause severe complications such as strictures, Barrett's esophagus, and esophageal adenocarcinoma. ${ }^{7}$ Thus, GERD is considered a major health concern in most countries.

In approximately $33-50 \%$ of patients with typical GERD symptoms, endoscopic examination reveals distal esophageal abnormalities such as erosions or ulcers, whereas the remaining patients do not show any endoscopic abnormalities. ${ }^{8,9}$ Thus, GERD is categorized into 2 subtypes based on endoscopic findings: erosive reflux disease (ERD) and non-erosive reflux disease (NERD). However, a few patients show endoscopic evidence of esophageal mucosal erosions/ulcers typically indicating erosive esophagitis (EE), although they remain asymptomatic and are therefore categorized into an asymptomatic EE (AEE) group. To date, it is unclear why a few patients with EE present with symptoms and others do not.

Many studies have investigated the relationship between functional gastrointestinal disorder and psychological factors. ${ }^{10,11} \mathrm{~A}$ close relationship has been established between the brain and the gastrointestinal tract. For example, stress and emotions can affect gastrointestinal function, as well as the occurrence of gastrointestinal symptoms and disease. Likewise, the state of the gastrointestinal organs may affect a person's emotional status. Psychological factors may influence the severity of a functional gastrointestinal disorder by affecting the perception of pain through an action on the gut-brain axis-a concept that is also applicable to patients with GERD. Additionally, when psychological factors accompany this condition, the treatment of the functional gastrointestinal disorder becomes difficult, which contributes to poor outcomes. ${ }^{10}$ To date, a few studies describing GERD have shown that psychological factors, particularly anxiety and depression, play an important role in patients with GERD; however, the results of those studies have been inconsistent. ${ }^{12-17}$ Many studies have reported a significant association of anxiety with GERD; however anxiety was not associated with the risk of GERD in a 10-year follow-up study in Sweden. ${ }^{15}$ Furthermore, in 1 prospective observational cohort study, high anxiety levels were not associated with the number of reflux symptoms, but only showed a relation to the severity of some reflux symptoms such as retrosternal pain or burning. ${ }^{14}$ In addition, some studies failed to demonstrate a significant association between depression and GERD. ${ }^{12,14-16}$ Moreover, only a few studies have analyzed the differences in the effects of psychological factors among the subtypes of GERD. ${ }^{12,17}$

Given this background, we examined the risk factors, particularly the psychological factors, associated with the subtypes of GERD in Koreans who underwent a health check-up. A clear understanding of the association between GERD and psychological factors is useful for administering optimal treatment in subjects with GERD because psychological factors can exacerbate GERD symptoms, worsen treatment outcomes, and interfere with the quality of life.

\section{Materials and Methods}

\section{Subjects and Study Design}

We performed a retrospective cross-sectional study that included 27906 subjects who underwent upper gastrointestinal endoscopy and completed a symptom questionnaire on the same day for routine health checkup at the Seoul National University Hospital Healthcare System Gangnam Center between January 2008 and December 2011. A schematic protocol of the study design is illustrated in Figure 1. We excluded 932 subjects from the analysis based on the following criteria: a diagnosis of gastroesophageal carcinoma or active peptic ulcer based on upper gastrointestinal endoscopy findings, and a history of gastrectomy. In addition, we excluded the subjects with symptoms of functional gastrointestinal disorder other than reflux from the control group, which could lead to bias in the results of the study. Eventually, 19099 subjects were included in the study, and their clinical and endoscopic records were reviewed. The study protocol was approved by the ethics committee of Seoul National University Hospital (institutional review board No. H-1510-046-710). Written informed consent was obtained from all subjects before every procedure.

\section{Clinical and Laboratory Evaluations}

All subjects completed a structured, self-administered questionnaire about basic demographic characteristics, general health and lifestyle, medical history, and gastrointestinal symptoms. Height and body weight were measured using a digital scale. The body mass index (BMI) was calculated as weight $(\mathrm{kg}) /$ height $^{2}\left(\mathrm{~m}^{2}\right)$. Based 


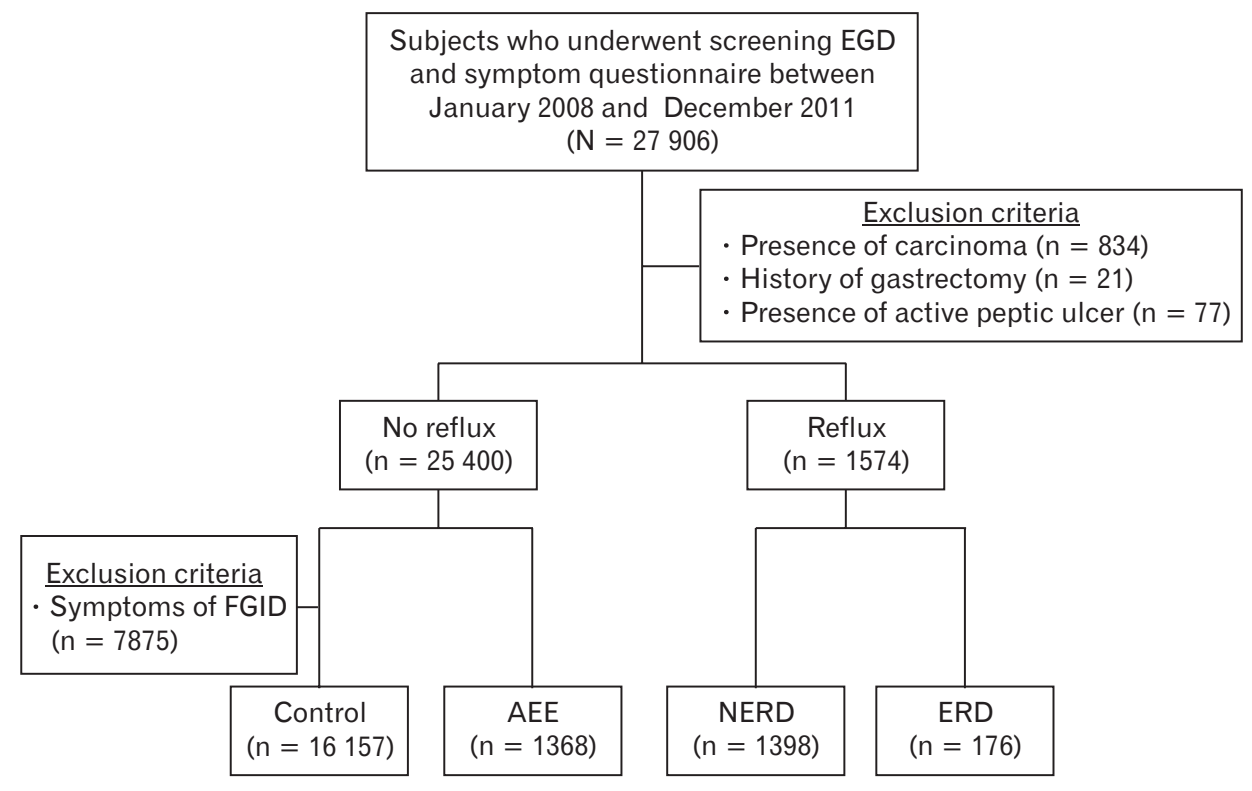

Figure 1. Study population. EGD, esophagogastroduodenoscopy; FGID, functional gastrointestinal disorder; AEE, asymptomatic erosive esophagitis; NERD, non-erosive reflux disease; ERD, erosive reflux disease. on smoking status, subjects were categorized as current smoker or non-current smoker (former or never smoker). Based on alcohol consumption, subjects were categorized as alcohol user or non-user (does not drink any alcohol). Laboratory evaluation was performed on the same day, including serum Helicobacter pylori IgG antibody test (H. pylori-EIA-Well kit; Radim, Rome, Italy), for determining the presence of $H$. pylori infection.

\section{Endoscopic Examinations}

All endoscopic examinations were performed by 17 boardcertified gastroenterologists. Conventional white light endoscopes (Olympus GIF-H260 and GIF-H290 series; Olympus Optical Co, Tokyo, Japan) were used for all procedures. In subjects who showed macroscopic findings of EE, atrophic gastritis, and intestinal metaplasia, the severity of the lesions was assessed and graded at each endoscopic examination. Atrophic gastritis was defined as endoscopically proven thinning of the gastric mucosa in the antrum or the body, with transparent submucosal blood vessels. Intestinal metaplasia was defined as ash-colored nodular change observed endoscopically.

\section{Definition of Gastroesophageal Reflux Disease and Its Subtypes}

Before undergoing the endoscopic examination, all subjects submitted themselves to a medical interview by physicians for the evaluation of gastrointestinal symptoms. The findings were recorded in a symptoms checklist. In the present study, based on the Montreal definition, we defined GERD as the presence of heart- burn (burning sensation in the retrosternal area) and/or acid regurgitation (perception of reflux of stomach contents into the mouth or the hypopharynx) occurring at least once a week. ${ }^{1}$ Among subjects with GERD, based on the Los Angeles classification of esophagitis, those with endoscopically proven esophageal mucosal erosions/ulcers were classified as the ERD group, and the remaining subjects were classified as the NERD group. Those with esophageal mucosal erosions/ulcers without reflux symptoms were categorized as the AEE group. Those without reflux symptoms and with normal endoscopic findings were categorized as the control group.

The study did not include the results of esophageal impedance$\mathrm{pH}$ monitoring tests or the response to proton pump inhibitors (PPIs). Therefore, subjects included in the NERD group may have been markedly heterogeneous, such as functional heartburn, acid hypersensitive esophagus, and non-acid hypersensitive esophagus. ${ }^{18,19}$ Patients with functional heartburn, in particular, were reported to have higher levels of anxiety than patients with GERD, and the inclusion of functional heartburn patients in the NERD group may exaggerate the potential association between NERD and psychological factors. ${ }^{14}$ Careful interpretation of the results is warranted.

\section{Evaluation of the State of Anxiety and Depression}

The State-Trait Anxiety Inventory (STAI) scale was used to assess the level of anxiety in all subjects. ${ }^{20}$ The STAI is a well-known psychological instrument comprising 2 self-report rating scales with 20 items each, for the measurement of 2 types of anxiety: state anxiety (how one feels at the moment, STAI-X1) and trait anxiety (how 
one generally feels, STAI-X2). Each item is rated between 1 and 4 depending on the frequency of target complaints (never, sometimes, often, and always), and overall scores are obtained by summing the ratings for the items (range, 20-80). We considered subjects showing moderate-to-severe state or trait anxiety by using a cut-off value of STAI-X1 $\geq 57$ or STAI-X2 $\geq 59$, respectively.

The depression status of subjects was evaluated using the Beck Depression Inventory (BDI) scale, one of the most commonly used self-report instruments designed to detect and measure the severity of depression in the general population. ${ }^{21}$ The BDI comprises of 21 items describing symptoms and attitudes to depression, and each item is rated between 0 and 3 in terms of intensity. The total score ranges between 0 and 63 - the higher the score, the greater the degree of depression. By using the cutoff value of 15 , subjects were classified as having no-to-mild depression $(\mathrm{BDI}<15$ ) or moderate-to-severe depression $(\mathrm{BDI} \geq 15)^{22}$

\section{Statistical Methods}

All data were expressed as means $\pm \mathrm{SD}$ or as numbers (percentages). The means of continuous variables were compared using one-way ANOVA, and categorical variables were compared using the chi-square test. Differences in anxiety or depression levels among GERD subtypes were analyzed using one-way ANOVA with Tukey's honestly significant difference post hoc analysis. Multinomial logistic regression analysis was used to evaluate the clinical characteristics of the subjects in the 4 groups. Univariate multinomial logistic regression was used to screen for important clinical parameters. Variables showing a $P$-value of $<0.05$ from univariate multinomial logistic regression were included in multiple multinomial logistic regression. ORs and 95\% CIs were used to estimate the association between various clinical and psychological characteristics and the GERD subtypes. A $P$-value of $<0.05$ was considered statistically significant. Statistical analyses were performed using R software, version 3.4.3 (R Foundation for Statistical Computing, Vienna, Austria). For multinomial logistic regression analysis, we used the Vector Generalized Additive Models package for $\mathrm{R}$.

\section{Results}

\section{Characteristics of the Study Subjects}

This study included 19099 subjects, among whom 1574 (8.2\%) had reflux symptoms. Subjects with reflux symptoms were classified into the ERD (176, 0.9\%) and NERD (1398, 7.3\%) groups ac- cording to the endoscopic findings of the gastroesophageal junction. Among 19099 subjects, 1368 (7.2\%) had endoscopically proven esophageal mucosal erosions/ulcers but did not have any reflux symptoms, and they were classified as the AEE group. The remaining subjects $(16157,84.6 \%)$ were classified as the control group. The baseline demographic and clinical characteristics of the study subjects are shown in Table 1. Subjects in the ERD and NERD groups were significantly younger than those in the control group $(45.8 \pm 10.9$ years and $47.0 \pm 11.5$ years vs $48.9 \pm 11.1$ years, $P<0.001$, respectively). The percentage of women was lower in the ERD and AEE groups than in the control group (14.2\% and $11.5 \%$ vs $37.3 \%, P<0.001$, respectively), but higher in the NERD group than in the control group $(42.4 \%$ vs $37.3 \%, P<$ 0.001). BMI was significantly higher in the ERD and AEE groups than in the control group $(25.5 \pm 3.2$ and $25.1 \pm 3.0$ vs $23.6 \pm 3.0$, $P<0.001$, respectively). When compared to the control group, the percentage of subjects with diabetes was significantly higher in the AEE group and significantly lower in the NERD group $(P<$ 0.001 , all). The percentage of current smokers and alcohol user was significantly higher in the ERD group and the NERD group than in the control group $(P<0.001$, all). The percentage of married subjects was significantly lower in the 2 subgroups showing reflux symptoms (ERD and NERD groups) than in the control group $(P<0.001)$. Compared with the control group, the percentage of those who used aspirin showed a statistically significant difference only in the AEE group $(P<0.001)$. The percentage of NSAIDs users was not significantly different from the control group in all 3 subtypes. The $H$. pylori seropositivity rates and incidence of endoscopic atrophic gastritis were significantly lower in the 3 subtypes than in the control group, whereas the incidence of intestinal metaplasia was significantly lower in the NERD group and AEE group than in the control group.

\section{Relationship Between Psychological Status and Gastroesophageal Reflux Disease}

The contribution of psychological factors associated with each subtype of GERD is shown in Figure 2. The level of state anxiety was significantly higher in the NERD group $(P<0.001)$ and the $\mathrm{ERD}$ group $(P=0.002)$ than in the control group, whereas the AEE group showed no significant difference from the control group. The level of trait anxiety was significantly different only in the NERD group among the subtypes of GERD $(P<0.001)$. Concerning depression level, there was a significant difference between the ERD and control $(P<0.001)$, NERD and control $(P<0.001)$, and the AEE and control groups $(P=0.001)$. In 


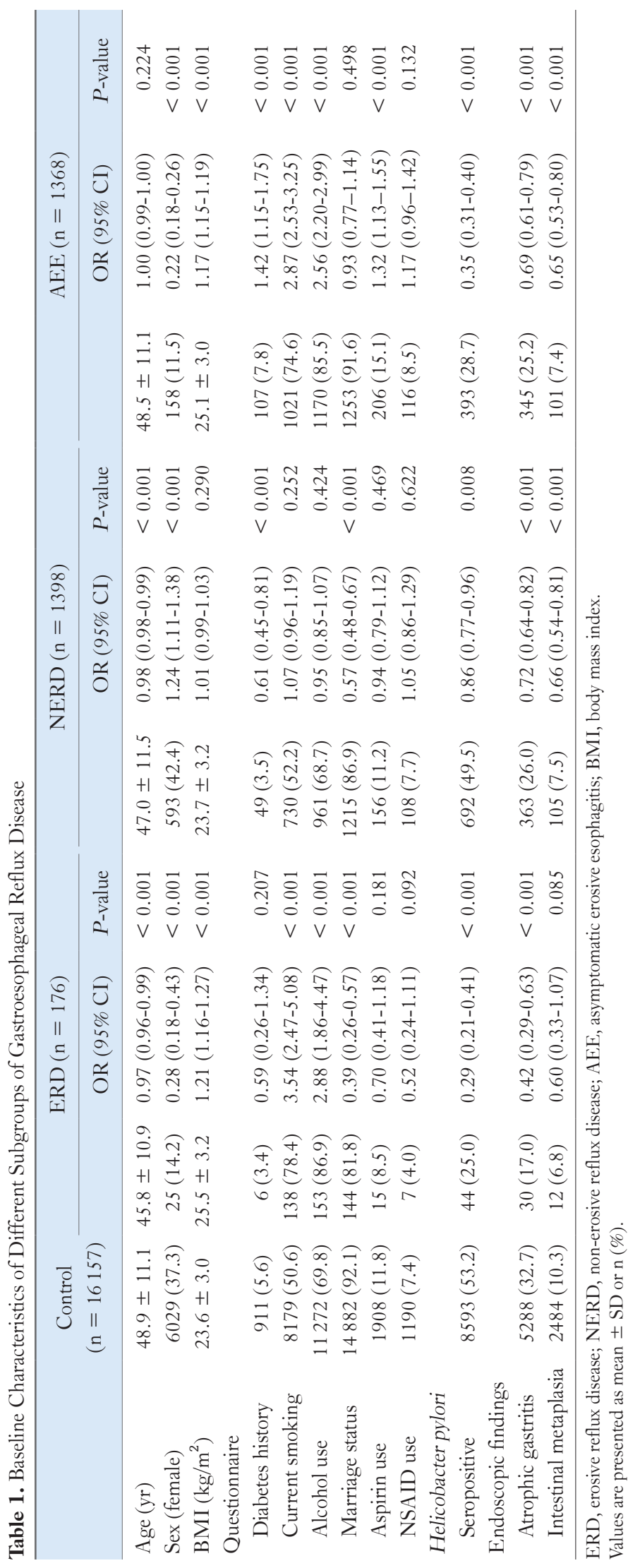



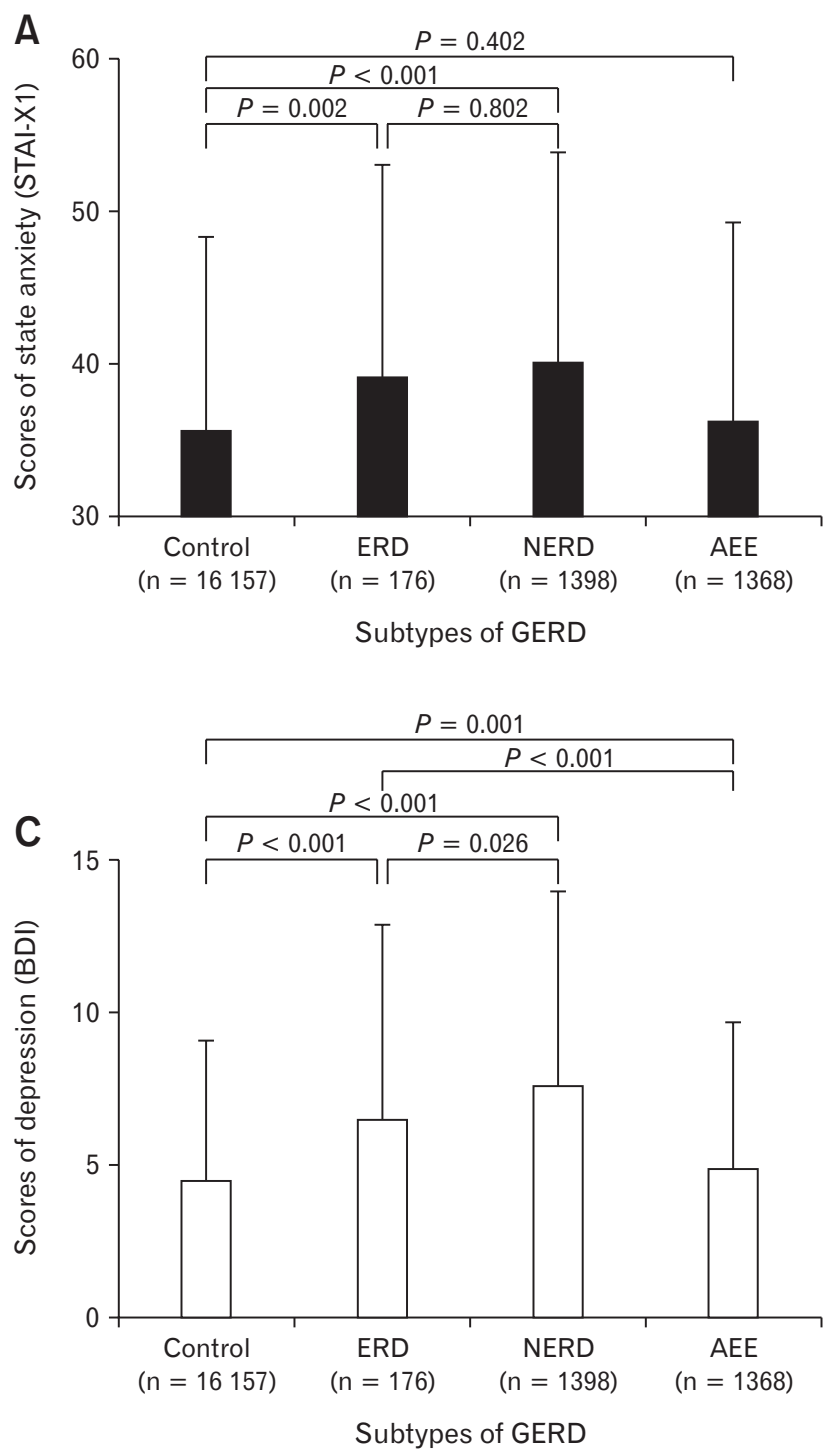

addition, significant differences were also observed among the 3 subtypes (NERD $>$ ERD $>$ AEE $>$ control). The percentage of subjects who exceeded the cutoff value for each psychological score (ie, those presenting with moderate-to-severe psychological factors) showed the same pattern as the aforementioned comparison using the mean value (data not shown).

\section{Factors Associated With Each Subtype of Gastroesophageal Reflux Disease}

The results of the multinomial logistic regression analysis are presented in Table 2. Female sex was observed to be an independent associated factor for NERD, and male sex for AEE. High $\mathrm{BMI}$ and current smoking were independently associated with all 3 subtypes of GERD. Alcohol use was associated only with the AEE

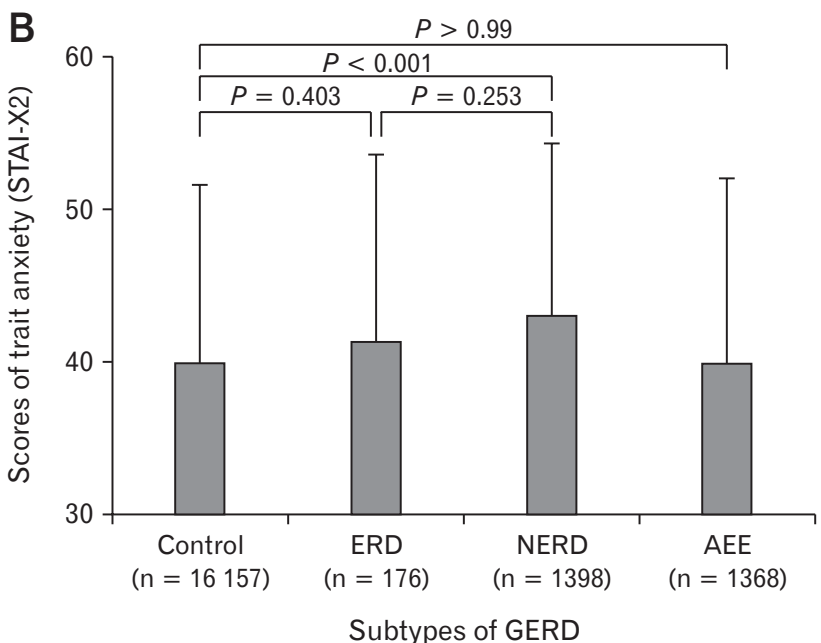

Figure 2. Psychological scores of different subtypes of gastroesophageal reflux disease (GERD). (A) Scores of state anxiety (State-Trait Anxiety Inventory [STAI]-X1) (B) Scores of trait anxiety (STAI-X2) (C) Scores of depression (Beck Depression Inventory [BDI]). ERD, erosive reflux disease; NERD, non-erosive reflux disease; AEE, asymptomatic erosive esophagitis.

group among the 3 subtypes of GERD. Marital status showed a significant association with ERD and NERD. The presence of diabetes mellitus showed a significant inverse correlation with NERD. An H. pylori-seropositive status and endoscopically proven atrophic gastritis also showed significant inverse correlations with the ERD and AEE subtypes, whereas the presence of intestinal metaplasia showed a significant inverse correlation with the NERD subtype.

Among the 3 GERD subtypes, NERD showed a significant association with all 3 psychological statuses examined in this study. NERD had a significant association with state anxiety (adjusted OR, 1.89; 95\% CI, 1.53-2.33) and trait anxiety (adjusted OR, 1.78; 95\% CI, 1.34-2.35). The NERD group also had a significant association with depression (adjusted OR, 221; 95\% CI, 1.75-2.80). However, only state anxiety and depression showed a significant 
Table 2. Multinomial Logistic Regression Analysis of Variables Associated With Subtypes of Gastroesophageal Reflux Disease

\begin{tabular}{|c|c|c|c|c|c|c|c|}
\hline & \multirow{2}{*}{ Control } & \multicolumn{2}{|l|}{ ERD } & \multicolumn{2}{|l|}{ NERD } & \multicolumn{2}{|l|}{$\mathrm{AEE}$} \\
\hline & & OR $(95 \% \mathrm{CI})$ & $P$-value & OR $(95 \% \mathrm{CI})$ & $P$-value & OR $(95 \% \mathrm{CI})$ & $P$-value \\
\hline Age & 1.00 & $1.01(0.99-1.02)$ & 0.495 & $1.00(0.99-1.00)$ & 0.157 & $1.00(1.00-1.01)$ & 0.524 \\
\hline Sex (female) & 1.00 & $0.68(0.39-1.18)$ & 0.168 & $1.42(1.20-1.69)$ & $<0.001$ & $0.38(0.31-0.48)$ & $<0.001$ \\
\hline BMI & 1.00 & $1.18(1.13-1.24)$ & $<0.001$ & $1.04(1.02-1.06)$ & $<0.001$ & $1.12(1.09-1.14)$ & $<0.001$ \\
\hline Current smoking & 1.00 & $2.28(1.47-3.55)$ & $<0.001$ & $1.37(1.18-1.59)$ & $<0.001$ & $1.46(1.26-1.70)$ & $<0.001$ \\
\hline Alcohol use & 1.00 & $1.44(0.88-2.34)$ & 0.144 & $0.96(0.83-1.10)$ & 0.531 & $1.25(1.05-1.49)$ & 0.012 \\
\hline Marriage status & 1.00 & $0.45(0.27-0.74)$ & 0.002 & $0.71(0.58-0.87)$ & $<0.001$ & $0.96(0.76-1.22)$ & 0.728 \\
\hline Diabetes mellitus & 1.00 & $0.51(0.22-1.18)$ & 0.116 & $0.65(0.48-0.87)$ & 0.004 & $1.13(0.91-1.41)$ & 0.269 \\
\hline Aspirin use & 1.00 & $0.80(0.43-1.49)$ & 0.480 & $1.00(0.80-1.25)$ & 0.993 & $1.11(0.91-1.36)$ & 0.310 \\
\hline NSAID use & 1.00 & $0.59(0.25-1.40)$ & 0.230 & $1.19(0.92-1.53)$ & 0.180 & $0.93(0.73-1.19)$ & 0.567 \\
\hline Helicobacter pylori seropositivity & 1.00 & $0.32(0.23-0.46)$ & $<0.001$ & $0.96(0.86-1.08)$ & 0.540 & $0.36(0.31-0.40)$ & $<0.001$ \\
\hline Atrophic gastritis & 1.00 & $0.55(0.35-0.87)$ & 0.010 & $0.88(0.76-1.02)$ & 0.084 & $0.80(0.69-0.93)$ & 0.003 \\
\hline Intestinal metaplasia & 1.00 & $1.10(0.58-2.11)$ & 0.763 & $0.79(0.64-0.99)$ & 0.038 & $0.84(0.67-1.06)$ & 0.147 \\
\hline State anxiety & 1.00 & $2.20(1.27-3.81)$ & 0.005 & $1.89(1.53-2.33)$ & $<0.001$ & $1.25(0.96-1.65)$ & 0.103 \\
\hline Trait anxiety & 1.00 & $1.07(0.44-2.59)$ & 0.882 & $1.78(1.34-2.35)$ & $<0.001$ & $1.27(0.83-1.96)$ & 0.270 \\
\hline Depression & 1.00 & $2.23(1.18-4.22)$ & 0.013 & $2.21(1.75-2.80)$ & $<0.001$ & $1.29(0.93-1.80)$ & 0.132 \\
\hline
\end{tabular}

ERD, erosive reflux disease; NERD, non-erosive reflux disease; AEE, asymptomatic erosive esophagitis; BMI, body mass index.

correlation with ERD, and psychological status did not show any correlation with AEE.

\section{Discussion}

In this study, we investigated the association between psychological status, such as anxiety and depression, and the clinical manifestations of GERD in the Korean population. After adjusting for various clinical factors, subjects in the ERD group showed higher state anxiety and depression levels than those in the control group. Subjects in the NERD group showed higher levels of all 3 psychological conditions (ie, state anxiety, trait anxiety, and depression) than those in the control group. Psychological factors were observed to be not associated with AEE. Thus, anxiety and depression levels were significantly higher in subjects with GERD, most notably in the NERD group.

Concerning the baseline characteristics, male sex was associated with the AEE group and the female sex was associated with the NERD group, which was in agreement with the results of previous studies. ${ }^{23-28}$ A high BMI was identified as a common associated factor among all 3 subgroups of GERD in this study. Prior studies have reported a positive correlation between BMI and disease prevalence in ERD and AEE. ${ }^{23,24,28}$ Obesity causes an increase in intragastric pressure, and a consequently elevated gastroesophageal pressure gradient leads to the development of hiatal hernia, which is known to be significantly associated with esophagitis. ${ }^{29}$ How- ever, although several studies have been performed, the association between NERD and BMI has not yet been conclusively established. $^{23,24,30}$ In this study, a $H$. pylori-seropositive status was inversely correlated with both $\mathrm{EE}$ with and without reflux symptoms, but not with NERD-findings that are in agreement with previous studies. $^{23,24,30,31}$

Approximately $50 \%$ of persons with typical reflux symptoms present with an associated endoscopically proven abnormality, and approximately $50 \%$ of those with typical reflux-related esophagitis are asymptomatic. ${ }^{25,31}$ This discrepancy between the patients' symptom perception and the degree of endoscopically identified mucosal injury suggests that other factors in addition to the pathogenesis of GERD may influence its clinical manifestations, and the psychological aspect is considered one such determinant.

In the current study, state and trait anxiety and depression levels were compared between controls and each subgroup of GERD. The results of this study demonstrated that the levels of both anxiety and depression were higher in subjects with GERD than in controls. A detailed analysis of the subtypes of GERD showed a significant association of state and trait anxiety and depression with NERD. Only state anxiety and depression showed a significant association with ERD. However, no significant difference was observed in the level of anxiety and depression in the AEE compared with the control group.

Previous studies that examined the association between GERD and psychological factors reported that anxiety was significantly as- 
sociated with GERD, and the results of this current study were in agreement with those findings. ${ }^{12-14,17,32}$ The association between the clinical manifestations of GERD and psychological factors such as anxiety and depression can be explained by the following mechanism. Psychological factors precede the manifestations of GERD. Certain psychological conditions such as anxiety may directly promote acid reflux by lowering the pressure of the lower esophageal sphincter, changing esophageal motility, or increasing gastric acid secretion. ${ }^{33,34}$ The mechanism by which psychological factors influence reflux symptoms has also been demonstrated in animal studies. Rats subjected to psychological stress showed disruption of the tight junctions of the esophageal epithelium with a consequent weakening/lowering of the barrier function of the esophageal mucosa, thereby increasing its vulnerability to reflux. ${ }^{35}$ Moreover, anxiety and depression can lead to hypochondriasis, which indirectly lowers the threshold of reflux perception and exaggerates the sensation of reflux symptoms compared to that observed in controls. ${ }^{36,37}$ In a previous study, anxiety and depression levels did not significantly affect the acid exposure time and the number of reflux episodes, although the severity of reflux symptoms showed a significant association with anxiety levels in patients with GERD. ${ }^{14}$ Conversely, reflux symptoms may cause anxiety and depression. Persistent reflux symptoms lead to distress and may precipitate anxiety and depression. Previous studies have shown that patients, particularly those with a poor response to PPIs, were more likely to show higher levels of anxiety and depression, and these partial PPI responders were most common in the NERD group among the subtypes of GERD. ${ }^{38}$ Therefore, the relationship between anxiety, depression, and GERD involves a complex interplay of various mechanisms, and a multidisciplinary approach is needed to understand this relationship.

In the present study, the prevalence of reflux symptoms was $8.2 \%$. This finding is comparable to that of previous studies that showed a change in the prevalence of GERD in Eastern Asia from $5.2 \%$ to $8.5 \%$ between 2005 and $2010 .^{23,39}$ The percentage of subjects with EE among those with GERD was approximately $11 \%$ (176 of 1574) in this study, which is lower than that in previous studies $(50 \%) .^{40,41}$ However, a nationwide multicenter prospective study that included subjects who underwent a health check-up across 40 health-care centers in Korea reported that only $14 \%$ of subjects with GERD showed endoscopic evidence of EE. ${ }^{23}$ This finding could be explained by the hypothesis that EE may have healed in a few patients owing to the widespread use of PPIs, and the percentage of patients with ERD may have been reduced because these patients have been reclassified into the NERD group. ${ }^{42}$
Notably, the subjects included in our study belonged to a high socioeconomic group, voluntarily participated in medical check-ups, and were more interested in and concerned about their health status, and also had better access to medical care than the general population. Thus, the study subjects may have been more frequently exposed to various drugs including PPIs. The lower percentage of patients belonging to the AEE subtype in this study than in previous studies could be attributed to this fact.

The strengths of this study include the following: (1) The large sample size ensured the robustness of our results. (2) The physician filled the questionnaire administered to all subjects for the assessment of reflux symptoms, which ensured the objectivity and completeness of the symptom evaluation. (3) The analyzed data included findings of endoscopic examinations; thus, we could evaluate the associations of the psychological factors separately and accurately in each subtype of GERD. (4) The STAI was able to assess both the acute and chronic anxiety levels including 2 types of anxiety (trait or chronic anxiety that reflects a person's permanent characteristics and state or acute anxiety that reflects a recent state). ${ }^{43}$

The limitations of this study are as follows: (1) As this was a cross-sectional study, we could not establish causal relationships between psychological factors and reflux symptoms. Further prospective longitudinal studies are warranted to elucidate the pattern of causality. (2) This study involved healthy persons who underwent health checkups and did not include the use of PPI for reflux symptoms. Thus, the characteristics of subjects included in this study may differ from those of patients visiting outpatient clinics. (3) This study lacks data about treatment with current anxiolytics or antidepressants. It has been shown that both tricyclic antidepressants, which reduce lower esophageal sphincter pressure, and selective serotonin reuptake inhibitors, which impair esophageal motility, can cause reflux episodes. Benzodiazepines, which are widely used as anti-anxiety medications, can reduce the pain threshold and affect the reflux perception. ${ }^{32}$ Therefore, it is difficult to definitively conclude whether the presence of reflux symptoms is related to anxiety and depressive symptoms themselves or to the use of specific psychiatric medications. (4) We did not evaluate the interobserver variability in the endoscopic diagnosis of $\mathrm{EE}$, atrophic gastritis, and intestinal metaplasia; however, the 17 gastroenterologists who performed the endoscopic examinations in this study were all board-certified experts with a minimum experience of 10000 upper endoscopies.

In conclusion, this large cross-sectional study demonstrated that levels of anxiety and depression were higher in subjects with GERD than in controls. Notably, subjects with NERD showed 
higher levels of anxiety and depression. A multidisciplinary approach to assess and manage these psychological factors is needed for the treatment of GERD.

\section{Financial support: None.}

\section{Conflict of interest: None.}

Author contributions: Ji Min Choi and Jong In Yang collected the cases, analyzed and interpreted the data, and wrote a manuscript; Seung Joo Kang designed, organized, and mediated the present study design, and collected cases and supervised the manuscript; Yoo Min Han, Jooyoung Lee, and Changhyun Lee contributed to the conception and the design of the study and to the collection, analysis, and interpretation of data; Su Jin Chung and Dae Hyun Yoon contributed to the conception and the design of the study, and to the interpretation of data; Boram Park analyzed and interpreted the data; and Yong Sung Kim contributed to the conception and the design of the study, analyzed and interpreted the data and supervised the manuscript.

\section{References}

1. Vakil N, van Zanten SV, Kahrilas P, Dent J, Jones R; Global Consensus Group. The montreal definition and classification of gastroesophageal reflux disease: a global evidence-based consensus. Am J Gastroenterol 2006;101:1900-1920.

2. Eusebi LH, Ratnakumaran R, Yuan Y, Solaymani-Dodaran M, Bazzoli F, Ford AC. Global prevalence of, and risk factors for, gastro-oesophageal reflux symptoms: a meta-analysis. Gut 2018;67:430-440.

3. Hunt R, Armstrong D, Katelaris P, et al. World gastroenterology organisation global guidelines: GERD global perspective on gastroesophageal reflux disease. J Clin Gastroenterol 2017;51:467-478.

4. Henke CJ, Levin TR, Henning JM, Potter LP. Work loss costs due to peptic ulcer disease and gastroesophageal reflux disease in a health maintenance organization. Am J Gastroenterol 2000;95:788-792.

5. Liker H, Hungin P, Wiklund I. Managing gastroesophageal reflux disease in primary care: the patient perspective. J Am Board Fam Pract 2005;18:393-400.

6. Min YW, Shin YW, Cheon GJ, et al. Recurrence and its impact on the health-related quality of life in patients with gastroesophageal reflux disease: a prospective follow-up analysis. J Neurogastroenterol Motil 2016;22:86-93.

7. Lundell LR, Dent J, Bennett JR, et al. Endoscopic assessment of oesophagitis: clinical and functional correlates and further validation of the Los Angeles classification. Gut 1999;45:172-180.

8. Fujiwara Y, Higuchi K, Shiba M, et al. Differences in clinical characteristics between patients with endoscopy-negative reflux disease and erosive esophagitis in Japan. Am J Gastroenterol 2005;100:754-758.

9. Labenz J, Jaspersen D, Kulig M, et al. Risk factors for erosive esophagitis: a multivariate analysis based on the ProGERD study initiative. Am J Gastroenterol 2004;99:1652-1656.

10. Van Oudenhove L, Crowell MD, Drossman DA, et al. Biopsychosocial aspects of functional gastrointestinal disorders. Gastroenterology 2016;150:1355-1367, e2.

11. Lee SP, Sung IK, Kim JH, Lee SY, Park HS, Shim CS. The effect of emotional stress and depression on the prevalence of digestive diseases. J Neurogastroenterol Motil 2015;21:273-282.

12. Kim JY, Kim N, Seo PJ, et al. Association of sleep dysfunction and emotional status with gastroesophageal reflux disease in Korea. J Neurogastroenterol Motil 2013;19:344-354.

13. Jansson C, Nordenstedt H, Wallander MA, et al. Severe gastro-oesophageal reflux symptoms in relation to anxiety, depression and coping in a population-based study. Aliment Pharmacol Ther 2007;26:683-691.

14. Kessing BF, Bredenoord AJ, Saleh CM, Smout AJ. Effects of anxiety and depression in patients with gastroesophageal reflux disease. Clin Gastroenterol Hepatol 2015;13:1089-1095, e1.

15. Aro P, Talley NJ, Johansson SE, Agréus L, Ronkainen J. Anxiety is linked to new-onset dyspepsia in the swedish population: a 10-year followup study. Gastroenterology 2015;148:928-937.

16. On ZX, Grant J, Shi Z, et al. The association between gastroesophageal reflux disease with sleep quality, depression, and anxiety in a cohort study of Australian men. J Gastroenterol Hepatol 2017;32:1170-1177.

17. Oh JH, Kim TS, Choi MG, et al. Relationship between psychological factors and quality of life in subtypes of gastroesophageal reflux disease. Gut Liver 2009;3:259-265.

18. Giacchino M, Savarino V, Savarino E. Distinction between patients with non-erosive reflux disease and functional heartburn. Ann Gastroenterol 2013;26:283-289.

19. Savarino E, Zentilin P, Savarino V. NERD: an umbrella term including heterogeneous subpopulations. Nat Rev Gastroenterol Hepatol 2013;10:371-380.

20. Julian LJ. Measures of anxiety: state-trait anxiety inventory (STAI), beck anxiety inventory (BAI), and hospital anxiety and depression scale-anxiety (HADS-A). Arthritis Care Res (Hoboken) 2011;63(suppl 11):S467S472.

21. Beck AT, Ward CH, Mendelson M, Mock J, Erbaugh J. An inventory for measuring depression. Arch Gen Psychiatry 1961;4:561-571.

22. Beck AT, Steer RA, Carbin MG. Psychometric properties of the beck depression inventory: twenty-five years of evaluation. Clin Psychol Rev 1988;8:77-100.

23. Kim N, Lee SW, Cho SI, et al. The prevalence of and risk factors for erosive oesophagitis and non-erosive reflux disease: a nationwide multicentre prospective study in Korea. Aliment Pharmacol Ther 2008;27:173-185.

24. Dore MP, Pes GM, Bassotti G, Farina MA, Marras G, Graham DY. Risk factors for erosive and non-erosive gastroesophageal reflux disease and barrett's esophagus in nothern sardinia. Scand J Gastroenterol 2016;51:1281-1287.

25. Lee D, Lee KJ, Kim KM, Lim SK. Prevalence of asymptomatic erosive esophagitis and factors associated with symptom presentation of erosive 
esophagitis. Scand J Gastroenterol 2013;48:906-912.

26. Cho JH, Kim HM, Ko GJ, et al. Old age and male sex are associated with increased risk of asymptomatic erosive esophagitis: analysis of data from local health examinations by the Korean national health insurance corporation. J Gastroenterol Hepatol 2011;26:1034-1038.

27. Wang PC, Hsu CS, Tseng TC, et al. Male sex, hiatus hernia, and Helicobacter pylori infection associated with asymptomatic erosive esophagitis. J Gastroenterol Hepatol 2012;27:586-591.

28. Wang FW, Tu MS, Chuang HY, Yu HC, Cheng LC, Hsu PI. Erosive esophagitis in asymptomatic subjects: risk factors. Dig Dis Sci 2010;55:1320-1324.

29. de Vries DR, van Herwaarden MA, Smout AJ, Samsom M. Gastroesophageal pressure gradients in gastroesophageal reflux disease: relations with hiatal hernia, body mass index, and esophageal acid exposure. Am J Gastroenterol 2008;103:1349-1354.

30. Minatsuki C, Yamamichi N, Shimamoto T, et al. Background factors of reflux esophagitis and non-erosive reflux disease: a cross-sectional study of 10,837 subjects in Japan. PLoS One 2013;8:e69891.

31. Lei WY, Yu HC, Wen SH, et al. Predictive factors of silent reflux in subjects with erosive esophagitis. Dig Liver Dis 2015;47:24-29.

32. Martín-Merino E, Ruigómez A, García Rodríguez LA, Wallander MA, Johansson S. Depression and treatment with antidepressants are associated with the development of gastro-oesophageal reflux disease. Aliment Pharmacol Ther 2010;31:1132-1140.

33. Avidan B, Sonnenberg A, Giblovich H, Sontag SJ. Reflux symptoms are associated with psychiatric disease. Aliment Pharmacol Ther
2001;15:1907-1912.

34. Johnston BT. Stress and heartburn. J Psychosom Res 2005;59:425-426.

35. Farre R, De Vos R, Geboes K, et al. Critical role of stress in increased oesophageal mucosa permeability and dilated intercellular spaces. Gut 2007;56:1191-1197.

36. Noyes R Jr. The relationship of hypochondriasis to anxiety disorders. Gen Hosp Psychiatry 1999;21:8-17.

37. Kamolz T, Velanovich V. Psychological and emotional aspects of gastroesophageal reflux disease. Dis Esophagus 2002;15:199-203.

38. Kimura Y, Kamiya T, Senoo K, et al. Persistent reflux symptoms cause anxiety, depression, and mental health and sleep disorders in gastroesophageal reflux disease patients. J Clin Biochem Nutr 2016;59:71-77.

39. Jung HK. Epidemiology of gastroesophageal reflux disease in Asia: a systematic review. J Neurogastroenterol Motil 2011;17:14-27.

40. Dent J, El-Serag HB, Wallander MA, Johansson S. Epidemiology of gastro-oesophageal reflux disease: a systematic review. Gut 2005;54:710717.

41. DeVault KR, Castell DO; American college of Gastroentetology. Updated guidelines for the diagnosis and treatment of gastroesophageal reflux disease. Am J Gastroenterol 2005;100:190-200.

42. Hershcovici T, Fass R. Nonerosive reflux disease (NERD) - an update. J Neurogastroenterol Motil 2010;16:8-21.

43. Shek DT. Reliability and factorial structure of the Chinese version of the State-Trait Anxiety Inventory. J Psychopathol Behav Assess 1988;10:303317. 